\title{
An evaluation of BRCA1 and BRCA2 founder mutations penetrance estimates for breast cancer among Ashkenazi Jewish women
}

\author{
Monica R. McClain, PhD ${ }^{1}$, Katherine L. Nathanson, $M D^{2}$, Glenn E. Palomaki, BS ${ }^{1}$, and James E. Haddow, $M D^{1}$
}

\begin{abstract}
Purpose: Three founder mutations in BRCA1 or BRCA2 genes increase breast cancer risk among Ashkenazi Jewish women. Reported estimates of the magnitude of this risk vary widely. We describe an integrated approach for assessing the plausibility of these estimates. Methods: Our approach integrates four epidemiologic parameters: (1) the proportion of all breast cancer cases with a founder mutation, (2) the proportion of women that carry one of these mutations, (3) the proportion of women with a mutation that develops cancer, and (4) the number of women who will develop cancer, regardless of mutation status. We then assess the published estimates of the proportion of Ashkenazi Jewish women with a mutation that develops cancer in the context of the other three parameters. Results: Penetrance for the founder mutations by ages 40, 50, and 70 are approximately $7 \%, 20 \%$, and $40 \%$, respectively. In two of the four published studies that evaluated at least two of the four parameters, penetrance estimates were internally consistent with the other three parameters and were also consistent with our consensus estimate. The third study had incomplete data. In the fourth study, the penetrance estimate was not internally consistent with the other three parameters, nor was it consistent with the consensus estimate. Conclusions: The four epidemiologic parameters are interdependent and can be used to test the plausibility of any one parameter. Based on the range of breast cancer penetrance estimates for BRCA1 and BRCA2 founder mutations derived by our approach, recently reported penetrance estimates appear to be overestimated. Genet
\end{abstract} Med 2005:7(1):34-39.

Key Words: breast cancer, genetics, public health, BRCA mutations, Ashkenazi Jewish

Three specific mutations account for approximately $85 \%$ of the mutations found in the BRCA1 (185delAG and 5382insC) and BRCA2 (6174delT) genes among the Ashkenazi Jewish population. ${ }^{1}$ The prevalence of these three mutations is approximately 10 times that of all $B R C A 1$ and $B R C A 2$ mutations among the general U.S. population. ${ }^{2-13}$ The risk of developing breast and ovarian cancer is significantly increased in women who carry one (or more) of these three founder mutations, although the magnitude of risk is less certain.

Four epidemiologic parameters must be known to accurately determine the burden of these three mutations in breast cancer cases among Ashkenazi Jewish women: (1) the proportion of all breast cancer cases associated with one of the three mutations (clinical sensitivity), (2) the proportion of women

\footnotetext{
From ${ }^{1}$ Foundation for Blood Research, Scarborough, Maine; and ${ }^{2}$ University of Pennsylvania, Department of Medicine, Philadelphia, Pennsylvania.

Monica R. McClain, PhD, Foundation for Blood Research, PO Box 190, 69 US Route 1, Scarborough, $M E$.

Received: July 14, 2004.

Accepted: September 27, 2004.
}

DOI: 10.1097/01.GIM.0000151156.14983.08 with one of the three mutations that will develop breast cancer (penetrance), (3) the prevalence of these three mutations among all women (carrier rate), and (4) the number of women that will develop breast cancer by a given age, regardless of mutation status (cumulative incidence).

To date, individual studies have derived estimates for between one and three of these epidemiologic parameters. ${ }^{2-7,14-17}$ Their estimates have not always been consistent, either with each other or internally. In order to be internally consistent, any given parameter estimate reported by a study needs to fall within a range of values that is determined by the remaining three epidemiologic parameter values. The present analysis is based on recognition that estimates for these four epidemiologic parameters are interdependent and can be evaluated and refined by entering them into a mathematical equation. To our knowledge, this is the first attempt to reconcile estimates of these parameters using such an integrated approach.

\section{MATERIALS AND METHODS}

The four interdependent epidemiologic parameters used in our approach are described here and in Table 1. 
Table 1

Ranges of published values for four epidemiologic parameters that characterize the relationship between $B R C A 1$ and $B R C A 2$ founder mutations and breast cancer among U.S. Ashkenazi Jewish women

\begin{tabular}{|c|c|c|c|}
\hline Epidemiologic parameter and definition & By age 40 & By age 50 & By age 70 \\
\hline Cumulative incidence (\%) & 0.5 & 2.3 & 10.1 \\
\hline \multicolumn{4}{|c|}{ Proportion of women that will develop breast cancer by a given age $e^{21}$} \\
\hline Clinical sensitivity $(\%)$ & $28-43$ & $16-22$ & $6.7-11.6$ \\
\hline \multicolumn{4}{|c|}{$\begin{array}{l}\text { Proportion of women with breast cancer by a given age that has a } B R C A 1 \text { or } B R C A 2 \text { founder } \\
\text { mutation }{ }^{2,14-16}\end{array}$} \\
\hline \multicolumn{4}{|l|}{ Penetrance $(\%)$} \\
\hline \multicolumn{4}{|c|}{$\begin{array}{l}\text { Proportion of women with a } B R C A 1 \text { or } B R C A 2 \text { founder mutation that develops breast } \\
\text { cancer by a given age }\end{array}$} \\
\hline BRCA1 alone $\mathrm{e}^{2,7,14-17}$ & $7-21$ & $18-39$ & $36-69$ \\
\hline$B R C A 2$ alone $e^{2,7,14-17}$ & $1-17$ & $6-34$ & $26-74$ \\
\hline$B R C A 1$ and $B R C A 2^{a}$ & $5-20$ & $15-35$ & $30-70$ \\
\hline \multicolumn{4}{|l|}{ Carrier rate } \\
\hline \multicolumn{4}{|c|}{ The rate of women with a $B R C A 1$ or $B R C A 2$ founder mutation in the general population } \\
\hline 185delAG $(B R C A 1)^{2,3,5-7}$ & & 1 in 92 to 1 in 130 & \\
\hline 5382 insC $(B R C A 1)^{2,3,5,7}$ & & 1 in 250 to 1 in 833 & \\
\hline 6174delT $(B R C A 2)^{2-5,7}$ & & 1 in 66 to 1 in 111 & \\
\hline Three mutations combined & & 1 in 33 to 1 in 56 & \\
\hline
\end{tabular}

${ }^{a}$ Approximate weighted average of the combined penetrance for BRCA1 and BRCA2 founder mutations.

\section{(1) Proportion of Ashkenazi Jewish women with breast cancer that has one of the three BRCA1 or BRCA2 founder mutations (clinical sensitivity)}

Direct estimates have been made for the proportion of population-based breast cancer cases at various ages associated with these mutations. ${ }^{2,14-16}$ The range of these estimates is reported in Table 1 . The estimates of clinical sensitivity are heterogeneous. Therefore, rather than providing a summary estimate in our integrated approach, we provide a sensitivity analysis over the range of estimates. The sensitivity analysis is appropriate when there is not consensus about what value should be assigned to a given parameter.

\section{(2) Proportion of women with one of the three BRCA1 or BRCA2 founder mutations that develops breast cancer (penetrance)}

Penetrance estimates for founder mutations have been reported for the Ashkenazi Jewish population., ${ }^{2,14-17}$ These estimates were derived indirectly, using a variety of methodologies. Table 1 shows that these point estimates cover a broad range. Some of the corresponding confidence intervals are also wide, indicating that the estimates are not precise. For these reasons, we include a sensitivity analysis for this variable in our integrated approach. The risk of breast cancer for BRCA1 founder mutation carriers is likely to be different than that for $B R C A 2$ founder mutation carriers. The 6174delT mutation in $B R C A 2$ falls within the ovarian cancer cluster region. There is evidence to suggest that carriers of mutations in this region have a decreased risk of breast cancer compared with other mutation carriers. ${ }^{18,19}$ Three studies that provide mutation sta- tus for all three founder mutations in women with breast cancer report that the two BRCA1 founder mutations (185delAG, 5382insC) represent between $64 \%$ and $72 \%$ of the three founder mutations. ${ }^{14-16} \mathrm{~A}$ weighted, combined penetrance estimate for all three founder mutations up to ages 40,50 , and 70 is used in our approach. Ages 40 and 50 were selected to show the contribution of founder mutations to early onset breast cancer. Age 70 was selected because all studies give a penetrance estimate to at least this age.

\section{(3) Proportion of women with one of the three BRCA1 and BRCA2 founder mutations in the general Ashkenazi Jewish population (carrier rate)}

This proportion has been directly estimated by several studies $^{2-7}$ and can be expressed as a carrier rate (e.g., $2.0 \%=1$ in 50). Table 1 shows the range of these estimates for each mutation, along with a consensus estimate range for all three.

\section{(4) Cumulative incidence of breast cancer to age 70 among Ashkenazi Jewish women}

There are no published population-based estimates available that report the cumulative incidence of breast cancer specifically for U.S. Ashkenazi Jewish women. An estimate of breast cancer incidence among Israeli Jews born in Europe or North America $\left(8.17 \%, 1988-1992^{20}\right)$ is lower than that in U.S. Caucasian women $\left(8.96 \%, 1993-1995^{21}\right)$. There is evidence to suggest that there is little or no difference in breast cancer risk between Ashkenazi Jewish women and the overall general population of Caucasian women in the U.S. ${ }^{22-25}$ We used the Dev- 
Can software, ${ }^{21}$ which uses the age-conditional probabilities by 5 -year intervals, ${ }^{26}$ to estimate the proportions of a theoretical cohort of one million 20-year-old Caucasian women in the U.S. general population who will develop breast cancer by ages 40, 50, and 70. These proportions are used as surrogate estimates for Ashkenazi Jewish women. Twenty years of age is selected as the starting reference point because BRCA1 and $B R C A 2$ mutation testing is recommended almost exclusively for adults, and because the incidence of breast cancer before age 20 is nearly zero. Ages 40 and 50 were selected to show the contribution of founder mutations to early onset breast cancer. We selected 70 years of age because all of the studies report the proportion of women with a mutation that develops breast cancer at least up to this age.

Our integrated approach defines the relationship among the four epidemiologic parameters in a hypothetical cohort of one million 20-year-old Ashkenazi Jewish women followed to ages 40,50 , and 70 and is derived from Bayes theorem. This integrated approach is represented by the following equation:

$$
\text { Cumulative incidence }(\%)=((\mathrm{p} \times 1,000,000) / \mathrm{cs}) \times \mathrm{cf}
$$

where $\mathrm{p}=$ combined penetrance of three founder mutations $(\%), \mathrm{cs}=$ clinical sensitivity of founder mutations (\%), and cf $=$ carrier frequency (carrier rate expressed as a percentage, $\%$ ). Any three of these epidemiologic parameter estimates can be used to compute the fourth. This integrated approach is then applied to data from four studies that report estimates for the proportion of women with breast cancer and a founder mutation (clinical sensitivity), along with penetrance..$^{2,14-16}$

\section{RESULTS}

Table 2 shows a two-way sensitivity analysis for penetrance of the three BRCA1 and BRCA2 founder mutations and the proportion of breast cancer cases associated with one of these mutations in a theoretical cohort of one million 20-year-old Ashkenazi Jewish women, assuming a mutation carrier rate of 1 in 40. The upper and lower limits for proportions of breast cancer cases with a mutation (columns) and penetrance (rows) are taken from Table 1. The associated cumulative incidences (table entries) are then computed for each combination, according to our equation. For example, by age 70 years, with mutations present in $10 \%$ of cancer cases and a mutation penetrance of $40 \%$, the cumulative incidence of breast cancer is computed to be 100,000 out of 1,000,000 women (boxed entry in Table 1). Figure 1 shows how this calculation is made.

In order to focus on plausible combinations of the four epidemiologic parameters, we have bolded selected entries in Table 2 , when all parameters are internally consistent (e.g., the computed cumulative incidence based on the other parameters is within $10 \%$ of the cumulative incidence in U.S. Caucasian women). In the top section of Table 2, the data show that when between $30 \%$ and $40 \%$ of breast cancer cases by age 40 is associated with a founder mutation, the mutation carrier rate is 1 in 40 , and the cumulative incidence of breast cancer cases is $0.5 \%$ ( $\pm 10 \%)$, the penetrance of founder mutations must be approximately $6 \%$ to $8 \%$. Similarly, the bottom section of Table 2 shows that penetrance to age 70 must be between $30 \%$ and $50 \%$. Collectively, the three sections of Table 2 show that the

Table 2

Cumulative incidence of breast cancer among one million Ashkenazi Jewish women derived using the interrelationships among the proportion of women with breast cancer that has a BRCA1 or BRCA2 founder mutation, the penetrance of founder mutations, and the founder mutation carrier rate of 1 in 40

\begin{tabular}{|c|c|c|c|c|c|c|}
\hline \multirow[b]{2}{*}{ Penetrance (\%) } & \multicolumn{6}{|c|}{ Proportion of women with breast cancer by age 40} \\
\hline & $30 \%$ & $32 \%$ & $34 \%$ & $36 \%$ & $38 \%$ & $40 \%$ \\
\hline 6 & 5,000 & 4,688 & 4,412 & 4,167 & 3,947 & 3,750 \\
\hline 7 & 5,833 & 5,469 & 5,147 & 4,861 & 4,605 & 4,375 \\
\hline \multirow[t]{2}{*}{8} & 6,667 & 6,250 & 5,882 & 5,556 & 5,263 & 5,000 \\
\hline & \multicolumn{6}{|c|}{ Proportion of women with breast cancer by age 50} \\
\hline Penetrance (\%) & $16 \%$ & $18 \%$ & $20 \%$ & $22 \%$ & & \\
\hline 15 & 23,438 & 20,833 & 18,750 & 17,045 & & \\
\hline 20 & 31,250 & 27,778 & 25,000 & 22,727 & & \\
\hline 25 & 39,063 & 34,722 & 31,250 & 28,409 & & \\
\hline
\end{tabular}

Proportion of women with breast cancer by age 70

\begin{tabular}{|c|c|c|c|c|c|c|}
\hline Penetrance (\%) & $7 \%$ & $8 \%$ & $9 \%$ & $10 \%$ & $11 \%$ & $12 \%$ \\
\hline 30 & 107,000 & 94,000 & 83,000 & 75,000 & 68,000 & 62,000 \\
\hline 40 & 143,000 & 125,000 & 111,000 & 100,000 & 91,000 & 83,000 \\
\hline 50 & 179,000 & 156,000 & 139,000 & 125,000 & 114,000 & 104,000 \\
\hline
\end{tabular}

Bolded items represent plausible combinations of the four epidemiologic parameter estimates and the boxed item represents the combination used in Fig. 1 (see text). 


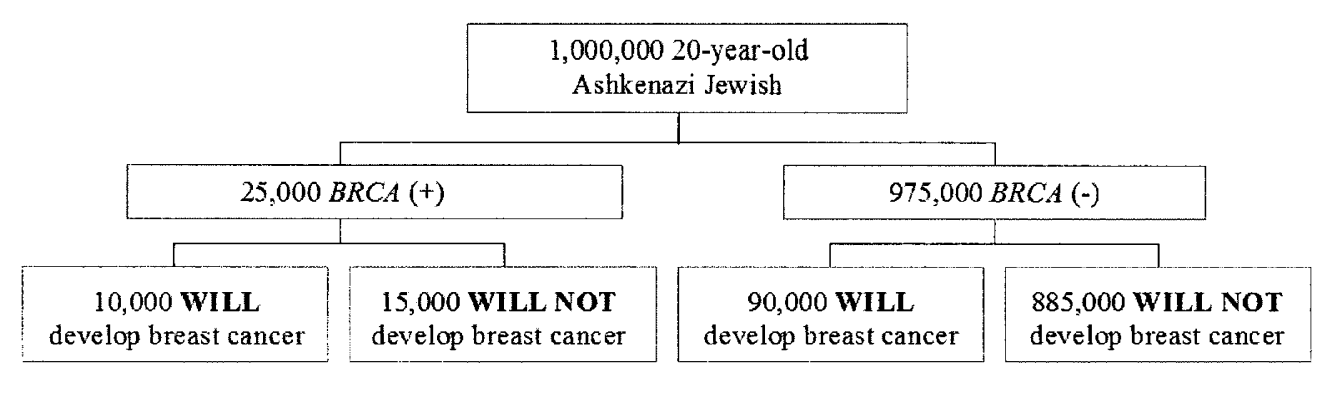

Cumulative Incidence $=10,000+90,000=100,000$

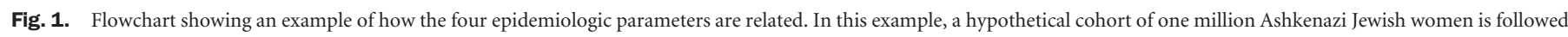

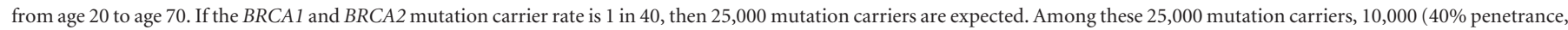

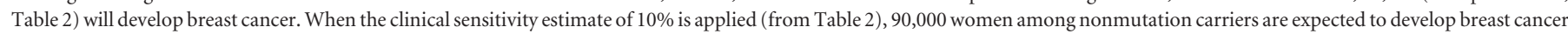
$(10,000 / 0.1)$. The cumulative incidence is, therefore, 100,000 or $10 \%$. Numbers in the remaining boxes in Fig. 1 are computed by subtraction.

proportion of breast cancer cases associated with a founder mutation decreases with age and the penetrance increases with age.

Although the mutation carrier rate of 1 in 40 among Ashkenazi Jewish women is commonly reported, the published estimates range from 1 in 33 to 1 in 56. If the higher carrier rate of 1 in 33 is entered into the analysis described above, the proportion of breast cancer cases by age 70 associated with a $B R C A 1$ or $B R C A 2$ founder mutation will lie between 9 and $12 \%$, and the mutation penetrance to age 70 will be between $30 \%$ and $40 \%$ (data not shown). If the lower carrier rate of 1 in 56 is used and between $7 \%$ and $12 \%$ of breast cancer cases are associated with a $B R C A 1$ or $B R C A 2$ founder mutation, then penetrance will lie between $40 \%$ and $70 \%$ (data not shown). Similar effects in clinical sensitivity and penetrance are found in breast cancer cases by ages 40 and 50 (data not shown).

Four studies report estimates for the proportion of Ashkenazi Jewish women with breast cancer and a founder mutation, along with penetrance of these mutations. ${ }^{2,14-16}$ The four epidemiologic parameters that are reported by and derived from these studies are summarized in Table 3. In two of these studies, the estimates reported are internally consistent. ${ }^{14,15}$
The calculated cumulative incidence estimates of 107,800 and 99,000 using data from the studies by Warner et al. ${ }^{14}$ and Satagopan et al. ${ }^{15}$, respectively, are within $7 \%$ of the SEER estimate $(101,200)$. The population investigated by Fodor et al. ${ }^{2}$ was also included in study by Satagopan et al. ${ }^{14}$; a low penetrance estimate was reported for 185delAG and 6174delT mutations (36\% to age 85 ), relative to other published penetrance estimates. The penetrance of 5382insC is not estimated, due to the low number of carriers. The proportion of women with breast cancer and a $B R C A 1$ or $B R C A 2$ founder mutation is also lower, at approximately $7 \%$. This study estimates the founder mutation carrier rate to be 1 in 45 . When these estimates are used in our integrated approach, a cumulative incidence of 114,300 breast cancer cases is expected. Surveillance, Epidemiology and End Results (SEER) estimates a cumulative incidence of 157,400 to age 85 . Thus, with a difference of over $40 \%$, these data are not likely to be internally consistent. Conversely, King et al. ${ }^{16}$ report a high penetrance estimate for $B R C A 1$ and $B R C A 2$ founder mutations (71\%) and a clinical sensitivity of $10 \%$. When these estimates are used in our integrated approach, along with a generally agreed upon founder mutation carrier rate of 1 in 40, the cumulative incidence is 177,500 by

Table 3

Summary of four epidemiologic parameters related to BRCA1 and BRCA2 mutations and breast cancer incidence by age 70 among Ashkenazi Jewish women

\begin{tabular}{|c|c|c|c|c|c|}
\hline Study & $\begin{array}{l}\text { Carrier rate } \\
(1 \text { in } N)\end{array}$ & $\begin{array}{c}\text { Clinical sensitivity } \\
(\%)\end{array}$ & $\begin{array}{c}\text { Penetrance-weighted } \\
\text { average }(\%)\end{array}$ & $\begin{array}{l}\text { Cumulative incidence } \\
\text { (calculated) }\end{array}$ & $\begin{array}{l}\text { Internally } \\
\text { consistent }\end{array}$ \\
\hline Fodor et al. ${ }^{a}$ & 45 & $6.7^{c}$ & $36^{d}$ & $119,400^{e}$ & Not likely \\
\hline Warner et al. & $40^{b}$ & 11.6 & 50 & 107,800 & Yes \\
\hline Satagopan et al. & $40^{b}$ & 10.1 & 40 & 99,000 & Yes \\
\hline King et al. & $40^{b}$ & 10.3 & 71 & 172,300 & No \\
\hline
\end{tabular}

${ }^{a}$ This study was included in the Satagopan et al. study.

${ }^{b}$ A mutation carrier rate of 1 in 40 is assumed.

${ }^{c}$ Clinical sensitivity was reported for 185 delAG, 5382insC, and 6174delT.

${ }^{d}$ Penetrance was reported for 185 delAG and 6174delT to age 85.

${ }^{e}$ Cumulative incidence is to age 85 and excludes cases with the 5382ins C mutation. 
age 70 . This is nearly $80 \%$ greater than the SEER estimate of about 100,000 . These data are clearly not internally consistent.

\section{DISCUSSION}

We developed the methodology described in this study to reconcile individual estimates of four epidemiologic parameters that have varying degrees of reliability, by integrating them into a single mathematical equation. Our study utilizes the inherent relationship among the four epidemiologic parameters. The estimate for any one parameter within the equation is constrained by the estimate (or range of estimates) for the other three. Based on published data, our integrated approach shows that, for Ashkenazi Jewish women, a combined BRCA1 and $B R C A 2$ founder mutation penetrance estimate for breast cancer by ages 40,50 , and 70 is most likely to lie between $6 \%$ and $8 \%, 15 \%$ and $22 \%$, and $30 \%$ and $50 \%$, respectively. These penetrance estimates may not be generalizable to all Jewish women with a BRCA1 or BRCA2 mutation that is not one of the three founder mutations. However, in a similar analysis conducted using epidemiologic parameter estimates for the general U. S. population, the penetrance to age 70 for all $B R C A 1$ and BRCA2 mutations was between $35 \%$ and $65 \% .{ }^{31}$

In our integrated approach, the penetrance estimates for $B R C A 1$ and BRCA2 founder mutations are combined. Differences in breast cancer risk have been reported for these three mutations. In addition, the 6174delT mutation in BRCA2 falls within the ovarian cancer cluster region. There is evidence to suggest that carriers of mutations in this region have a decreased risk of breast cancer compared with other mutation carriers. ${ }^{18,19}$ We have addressed these differences in risk by utilizing a weighted average. Alternatively, our integrated approach could also be used for each mutation or mutations in each gene. The clinical sensitivity and penetrance estimates used in this approach were derived from population-based cohorts. Estimates for these parameters obtained from families with multiple and/or early onset breast cancer cases are higher. Furthermore, there may be variations in penetrance due to environmental or gene modifiers. Although these penetrance variations cannot currently be measured, they are likely to be over-represented in the high-risk-based estimates.

There are two potential reasons why these penetrance and cumulative incidence estimates may be slightly underestimated. First, women carrying a BRCA1/2 mutation are at increased risk of dying at a younger age due to ovarian cancer and would therefore not develop breast cancer later in life (assuming they had not developed breast cancer before ovarian cancer). This is not likely to impact the estimates because the cumulative incidence of ovarian cancer is between five and nine times less than that of breast cancer, ${ }^{21}$ depending on the age cutoff selected. Second, mutation carriers are more likely to undergo risk reducing surgeries (mastectomy and/or oophorectomy). The impact of the surgeries depends on the proportion of mutation carriers that selects these preventive strategies, which is currently relatively low among mutation carriers in the United States. ${ }^{27-30}$
A limitation of this study is that there is no reliable estimate for the cumulative incidence of breast cancer among Ashkenazi Jewish women in the United States. There is no substantial evidence that this cumulative incidence is significantly different from that in the U.S. Caucasian population, which we have used as a surrogate estimate in our integrated approach. In fact, our analysis suggests that the cumulative incidence of breast cancer among Ashkenazi Jewish women is likely to be within $10 \%$ of that in the U.S. Caucasian population.

Our study finds that $B R C A 1$ and $B R C A 2$ founder mutation penetrance is not likely to be as high at any age as those recently reported by King et al. ${ }^{16}$ The penetrance estimates generated from our integrated approach are consistent with earlier published reports in this population. ${ }^{2,14,15}$ Our finding has implications for Ashkenazi Jewish women considering BRCA1 and $B R C A 2$ mutation testing and/or breast and ovarian cancer prevention strategies. These results also have a broader impact for public health genetics by better understanding the burden of these founder mutations on breast cancer in the U.S.

\section{ACKNOWLEDGMENTS}

We thank Muin Khoury, MD, PhD, Paula Yoon, $\mathrm{ScD}, \mathrm{MPH}$, and Linda A. Bradley, PhD, Office of Genomics and Disease Prevention, Centers for Disease Control and Prevention for their support of the ACCE project. We also thank Ingrid Hall, $\mathrm{PhD}$, Division of Cancer Prevention and Control, National Center for Chronic Disease Prevention and Health Promotion, Centers for Disease Control and Prevention for helping support the project. Support for this study was provided by a cooperative agreement (UR3/CCU319352) with the Centers for Disease Control and Prevention, Office of Genomics and Disease Prevention.

\section{References}

1. Frank TS, Deffenbaugh AM, Reid JE et al. Clinical Characteristics of Individuals With Germline Mutations in BRCA1 and BRCA2: Analysis of 10,000 Individuals. J Clin Oncol 2002;20:1480-1490.

2. Fodor FH, Weston A, Bleiweiss IJ et al. Frequency and carrier risk associated with common BRCA1 and BRCA2 mutations in Ashkenazi Jewish breast cancer patients. Am J Hum Genet 1998;63:45-51.

3. Hartge P, Struewing JP, Wacholder S, Brody LC, Tucker MA. The prevalence of common BRCA1 and BRCA2 mutations among Ashkenazi Jews. Am J Hum Genet 1999;64:963-970.

4. Oddoux C, Struewing JP, Clayton CM et al. The carrier frequency of the BRCA2 6174delT mutation among Ashkenazi Jewish individuals is approximately $1 \%$. Nat Genet 1996;14:188-190.

5. Roa BB, Boyd AA, Volcik K, Richards CS. Ashkenazi Jewish population frequencies for common mutations in BRCA1 and BRCA2. Nat Genet 1996;14:185-187.

6. Struewing JP, Abeliovich D, Peretz T et al. The carrier frequency of the BRCA1 185delAG mutation is approximately 1 percent in Ashkenazi Jewish individuals. Nat Genet Oct 1995;11:198-200.

7. Struewing JP, Hartge $P$, Wacholder $S$ et al. The risk of cancer associated with specific mutations of BRCA1 and BRCA2 among Ashkenazi Jews. N Engl J Med 1997;336: 1401-1408.

8. Prevalence and penetrance of BRCA1 and BRCA2 mutations in a population-based series of breast cancer cases: Anglian Breast Cancer Study Group. Br J Cancer 2000; 83:1301-1308.

9. Antoniou AC, Gayther SA, Stratton JF, Ponder BA, Easton DF. Risk models for familial ovarian and breast cancer. Genet Epidemiol 2000;18:173-190.

10. Antoniou AC, Pharoah PD, McMullan G et al. A comprehensive model for familial breast cancer incorporating BRCA1, BRCA2 and other genes. Br J Cancer 2002;86: $76-83$. 
11. Ford D, Easton DF, Peto J. Estimates of the gene frequency of BRCAl and its contribution to breast and ovarian cancer incidence. Am J Hum Genet 1995;57:1457-1462.

12. Peto J, Collins N, Barfoot R et al. Prevalence of BRCA1 and BRCA2 gene mutations in patients with early-onset breast cancer. J Natl Cancer Inst 1999;91:943-949.

13. Whittemore AS, Gong G, Itnyre J. Prevalence and contribution of BRCA1 mutations in breast cancer and ovarian cancer: results from three U.S. population-based casecontrol studies of ovarian cancer. Am J Hum Genet 1997;60:496-504.

14. Warner E, Foulkes W, Goodwin P et al. Prevalence and penetrance of BRCA1 and BRCA2 gene mutations in unselected Ashkenazi Jewish women with breast cancer. J Natl Cancer Inst 1999;91:1241-1247.

15. Satagopan JM, Offit K, Foulkes W et al. The lifetime risks of breast cancer in Ashkenazi Jewish carriers of BRCA1 and BRCA2 mutations. Cancer Epidemiol Biomarkers Prev 2001;10:467-473.

16. King MC, Marks JH, Mandell JB. Breast and ovarian cancer risks due to inherited mutations in BRCA1 and BRCA2. Science 2003;302:643-646.

17. Moslehi R, Chu W, Karlan B et al. BRCA1 and BRCA2 mutation analysis of 208 Ashkenazi Jewish women with ovarian cancer. Am J Hum Genet 2000;66:1259-1272.

18. Gayther SA, Mangion J, Russell P, et al. Variation of risks of breast and ovarian cancer associated with different germline mutations of the BRCA2 gene. Nat Genet 1997;15:103-105

19. Thompson D, Easton D. Variation in cancer risks, by mutation position, in BRCA2 mutation carriers. Am J Hum Genet 2001;68:410-419.

20. Cancer incidence in five continents [computer program]. Version 7. Lyon: International Agency for Research on Cancer; Publications No. 143, 1997.

21. DevCan software: Probability of developing or dying of cancer [computer program]. Version 5.1: Statistical Research and Application Branch, National Cancer
Institute; 2003. Available at: http://srab.cancer.gov/devcan. Accessed September 15, 2004.

22. Egan KM, Newcomb PA, Longnecker MP et al. Jewish religion and risk of breast cancer. Lancet 1996;347:1645-1646.

23. Feldman GE. Do Ashkenazi Jews have a higher than expected cancer burden? Implications for cancer control prioritization efforts. Isr Med Assoc J 2001;3:341-346.

24. Neuhausen SL. Ethnic differences in cancer risk resulting from genetic variation. Cancer 1999;86(suppl):2575-2582.

25. Toniolo PG, Kato I. Jewish religion and risk of breast cancer. Lancet 1996;348:760.

26. Fay MP, Pfeiffer R, Cronin KA, Le C, Feuer EJ. Age-conditional probabilities of developing cancer. Stat Med 2003;22:1837-1848.

27. Botkin JR, Smith KR, Croyle RT et al. Genetic testing for a BRCAl mutation: Prophylactic surgery and screening behavior in women 2 years post testing. Am J Med Genet 2003;118A:201-209.

28. Lerman C, Hughes C, Croyle RT et al. Prophylactic surgery decisions and surveillance practices one year following BRCA1/2 testing. Prev Med 2000;31:75-80.

29. Scheuer L, Kauff N, Robson M et al. Outcome of preventive surgery and screening for breast and ovarian cancer in BRCA mutation carriers. J Clin Oncol 2002;20: $1260-1268$.

30. Schwartz MD, Kaufman E, Peshkin BN et al. Bilateral prophylactic oophorectomy and ovarian cancer screening following BRCA1/BRCA2 mutation testing. J Clin Oncol 2003;21:4034-4041.

31. McClain MR, Palomaki GE, Nathanson KL, Haddon JE. Adjusting the estimated proportion of breast cancer cases associated with BRCA1 and BRCA2 mutations: Public health implications. Genet Med 2005;1:28-33. 